\title{
APPlying Knowledge: Evidence for and Regulation of Mobile Apps for Dermatologists
}

\author{
Cynthia X. Chan, BS; Orit Markowitz, MD
}

\section{PRACTICE POINTS}

- Physicians who are selecting an app for selfeducation or patient care should take into consideration the strength of the evidence supporting the app as well as the rigor of any approval process the app had to undergo.

- Only a minority of health-related apps are regulated by the government. This regulation has not kept up with the evolution of app software and may become more indirect.

Although the complexity of health-related applications (apps) has evolved, they have not been adequately regulated or monitored for quality. We review the primary literature behind and regulation of apps that impact dermatologists, with a focus on the 3 most prevalent dermatology-related apps used by dermatology residents in the United States: VisualDx, UpToDate, and Mohs Surgery Appropriate Use Criteria. These apps are widely utilized but have not undergone approval by the 3 main government agencies responsible for regulating mobile medical apps: the US Food and Drug Administration (FDA), Federal Trade Commission, and Office for Civil Rights. Health-related apps that target providers can be a valuable tool, but given their potential impact on human lives, they should be well regulated and evidence based. It is important that apps designed to assist in health care delivery are appropriately monitored and that physicians are aware of the rigor of review of the apps that they choose to use in clinical practice.

Cutis. 2020;106:76-78. $\bigcirc$ ince the first mobile application (app) was developed in the 1990s, apps have become increasingly integrated into medical practice and training. More than 5.5 million apps were downloadable in $2019,{ }^{1}$ of which more than 300,000 were health related. ${ }^{2}$ In the United States, more than $80 \%$ of physicians reported using smartphones for professional purposes in $2016 .^{3}$ As the complexity of apps and their purpose of use has evolved, regulatory bodies have not adapted adequately to monitor apps that have broad-reaching consequences in medicine.

We review the primary literature on PubMed behind health-related apps that impact dermatologists as well as the government regulation of these apps, with a focus on the 3 most prevalent dermatology-related apps used by dermatology residents in the United States: VisualDx, UpToDate, and Mohs Surgery Appropriate Use Criteria. This prevalence is according to a survey emailed to all dermatology residents in the United States by the American Academy of Dermatology (AAD) in 2019 (unpublished data).

\section{VisualDx}

VisualDx, which aims to improve diagnostic accuracy and patient safety, contains peer-reviewed data and more than 32,000 images of dermatologic conditions. The editorial board includes more than 50 physicians. It provides opportunities for continuing medical education credit, is used in more than 2300 medical settings, and costs $\$ 399.99$ annually for a subscription with partial

Ms. Chan is from the Geisel School of Medicine, Dartmouth College, Hanover, New Hampshire. Dr. Markowitz is from the Department of Dermatology, Mount Sinai Health System, New York, New York; the Department of Dermatology, SUNY Downstate Health Sciences University, Brooklyn; and the Department of Dermatology, New York Harbor Healthcare System, Brooklyn.

The authors report no conflict of interest.

Correspondence: Orit Markowitz, MD (omarkowitz@gmail.com)

doi:10.12788/cutis.0036 
features. Prior to the launch of the app in 2010, some health science professionals noted that the website version lacked references to primary sources. ${ }^{4}$ The same issue carried over to the app, which has evolved to offer artificial intelligence (AI) analysis of photographed skin lesions. However, there are no peer-reviewed publications showing positive impact of the app on diagnostic skills among dermatology residents or on patient outcomes.

\section{UpToDate}

UpToDate is a web-based database created in the early 1990s. A corresponding app was created around 2010. Both internal and independent research has demonstrated improved outcomes, and the app is advertised as the only clinical decision support resource associated with improved outcomes, as shown in more than 80 publications. ${ }^{5}$ UpToDate covers more than 11,800 medical topics and contains more than 35,000 graphics. It cites primary sources and uses a published system for grading recommendation strength and evidence quality. The data are processed and produced by a team of more than 7100 physicians as authors, editors, and reviewers. The platform grants continuing medical education credit and is used by more than 1.9 million clinicians in more than 190 countries. A 1-year subscription for an individual US-based physician costs $\$ 559$. An observational study assessed UpToDate articles for potential conflicts of interest between authors and their recommendations. Of the 6 articles that met inclusion criteria of discussing management of medical conditions that have controversial or mostly brand-name treatment options, all had conflicts of interest, such as naming drugs from companies with which the authors and/or editors had financial relationships. ${ }^{6}$

\section{Mohs Surgery Appropriate Use Criteria}

The Mohs Surgery Appropriate Use Criteria app is a free clinical decision-making tool based on a consensus statement published in 2012 by the AAD, American College of Mohs Surgery, American Society for Dermatologic Surgery Association, and American Society for Mohs Surgery. ${ }^{7}$ It helps guide management of more than 200 dermatologic scenarios. Critique has been made that the criteria are partly based on expert opinion and data largely from the United States and has not been revised to incorporate newer data. ${ }^{8}$ There are no publications regarding the app itself.

\section{Regulation of Health-Related Apps}

Health-related apps that are designed for utilization by health care providers can be a valuable tool. However, given their prevalence, cost, and potential impact on patient lives, these apps should be well regulated and researched. The general paucity of peer-reviewed literature demonstrating the utility, safety, quality, and accuracy of health-related apps commonly used by providers is a reflection of insufficient mobile health regulation in the United States.

There are 3 primary government agencies responsible for regulating mobile medical apps: the US Food and Drug Administration (FDA), Federal Trade Commission, and Office for Civil Rights. ${ }^{9}$ The FDA does not regulate all medical devices. Apps intended for use in the diagnosis, cure, mitigation, prevention, or treatment of a disease or condition are considered to be medical devices..$^{10}$ The FDA regulates those apps only if they are judged to pose more than minimal risk. Apps that are designed only to provide easy access to information related to health conditions or treatment are considered to be minimal risk but can develop into a different risk level such as by offering AI. ${ }^{11}$ Although the FDA does update its approach to medical devices, including apps and AI- and machine learning-based software, the rate and direction of update has not kept pace with the rapid evolution of apps. ${ }^{12}$ In 2019, the FDA began piloting a precertification program that grants long-term approval to organizations that develop apps instead of reviewing each app product individually. ${ }^{13}$ This decrease in premarket oversight is intended to expedite innovation with the hopeful upside of improving patient outcomes but is inconsistent, with the FDA still reviewing other types of medical devices individually.

For apps that are already in use, the Federal Trade Commission only gets involved in response to deceptive or unfair acts or practices relating to privacy, data security, and false or misleading claims about safety or performance. It may be more beneficial for consumers if those apps had a more stringent initial approval process. The Office for Civil Rights enforces the Health Insurance Portability and Accountability Act when relevant to apps.

Nongovernment agencies also are involved in app regulation. The FDA believes sharing more regulatory responsibility with private industry would promote efficiency. ${ }^{14}$ Google does not allow apps that contain false or misleading health claims, ${ }^{15}$ and Apple may scrutinize medical apps that could provide inaccurate data or be used for diagnosing or treating patients. ${ }^{16}$ Xcertia, a nonprofit organization founded by the American Medical Association and others, develops standards for the security, privacy, content, and operability of healthrelated apps, but those standards have not been adopted by other parties. Ultimately, nongovernment agencies are not responsible for public health and do not boast the government's ability to enforce rules or ensure public safety.

\section{Final Thoughts}

The AAD survey of US dermatology residents found that the top consideration when choosing apps was up-to-date and accurate information; however, the 3 most prevalent apps among those same respondents did not need government approval and are not required to contain up-to-date data or to improve clinical outcomes, similar to most other healthrelated apps. This discrepancy is concerning considering the 
increasing utilization of apps for physician education and health care delivery and the increasing complexity of those apps. In light of these results, the potential decrease in federal premarket regulation suggested by the FDA's precertification program seems inappropriate. It is important for the government to take responsibility for regulating healthrelated apps and to find a balance between too much regulation delaying innovation and too little regulation hurting physician training and patient care. It also is important for providers to be aware of the evidence and oversight behind the technologies they use for professional purposes.

\section{REFERENCES}

1. Clement J. Number of apps available in leading app stores as of 1st quarter 2020. Statista website. https://www.statista.com /statistics/276623/number-of-apps-available-in-leading-app-stores/. Published May 4, 2020. Accessed July 23, 2020.

2. mHealth App Economics 2017/2018. Current Status and Future Trends in Mobile Health. Berlin, Germany: Research 2 Guidance; 2018.

3. Healthcare Client Services. Professional usage of smartphones by doctors. Kantar website. https://www.kantarmedia.com/us /thinking-and-resources/blog/professional-usage-of-smartphones -by-doctors-2016. Published November 16, 2016. Accessed July 23, 2020.

4. Skhal KJ, Koffel J.VisualDx. J Med Libr Assoc. 2007;95:470-471.

5. UpToDate is the only clinical decision support resource associated with improved outcomes. UpToDate website. https://www.uptodate .com/home/research. Accessed July 29, 2020.

6. Connolly SM, Baker DR, Coldiron BM, et al. AAD/ACMS/ASDSA/ ASMS 2012 appropriate use criteria for Mohs micrographic surgery: a report of the American Academy of Dermatology, American College of Mohs Surgery, American Society for Dermatologic Surgery Association, and the American Society for Mohs Surgery. J Am Acad Dermatol. 2012;67:531-550.
7. Amber KT, Dhiman G, Goodman KW. Conflict of interest in online point-of-care clinical support websites. J Med Ethics. 2014;40:578-580.

8. Croley JA, Joseph AK, Wagner RF Jr. Discrepancies in the Mohs micrographic surgery appropriate use criteria. J Am Acad Dermatol. 2020;82:E55.

9. Mobile health apps interactive tool. Federal Trade Commission website. https://www.ftc.gov/tips-advice/business-center/guidance /mobile-health-apps-interactive-tool. Published April 2016. Accessed May 23, 2020.

10. Federal Food, Drug, and Cosmetic Act, 21 USC $\$ 321$ (2018).

11. US Food and Drug Administration. Examples of software functions for which the FDA will exercise enforcement discretion. https://www.fda.gov/medical-devices/device-software-functions -including-mobile-medical-applications/examples-software -functions-which-fda-will-exercise-enforcement-discretion. Updated September 26, 2019. Accessed July 29, 2020.

12. US Food and Drug Administration. Proposed regulatory framework for modifications to artificial intelligence/machine learning (AI/ML)-based software as a medical device (SaMD). https://www.fda .gov/downloads/MedicalDevices/DigitalHealth/Softwareasa MedicalDevice/UCM635052.pdf. Accessed July 23, 2020.

13. US Food and Drug Administration. Digital health software precertification (pre-cert) program. https://www.fda.gov /medical-devices/digital-health/digital-health-software -precertification-pre-cert-program. Updated July 18, 2019. Accessed July 23,2020 .

14. Gottlieb S. Fostering medical innovation: a plan for digital health devices. US Food and Drug Administration website. https://www.fda gov/news-events/fda-voices/fostering-medical-innovation-plan -digital-health-devices. Published June 15, 2017. Accessed July 23, 2020.

15. Restricted content: unapproved substances. Google Play website. https://play.google.com/about/restricted-content/unapproved -substances. Accessed July 23, 2020.

16. App store review guidelines. Apple Developer website. https://developer.apple.com/app-store/review/guidelines. Updated March 4, 2020. Accessed July 23, 2020. 